\title{
Effet de la fertilisation azotée et du stade de coupe sur le rendement, la composition chimique et la digestibilité in vitro des céréales plantes entières
}

\author{
GF Tremblay 1, D Pageau 1, J Surprenant 2, HV Petit 3, R Drapeau ${ }^{1}$ \\ 'Ferme de Recherches, Agriculture et Agro-alimentaire Canada, Normandin, QC, GOW 2EO; ?Centre \\ de recherche et de développement sur les sols et les grandes culfures, Agriculture et Agro-alimentaire \\ Canada, Ste-Foy, QC, G1V 2J3; 3Ferme de Recherches sur le mouton, Agriculture \\ et Agro-alimentaire Canada, La Pocatière, QC, GOR 1ZO, Canada
}

Les céréales sont occasionnellement utilisées comme fourrage. Le présent essai avait pour but de comparer le rendement, la composition chimique et la digestibilité des céréales (avoine, blé, orge et triticale) ayant reçu quatre niveaux de fertilisation azotée $(60,80,100$ et $120 \mathrm{~kg} \mathrm{~N} / \mathrm{ha}$ ) et récoltées à quatre stades de croissance (gonflement, épiaison, grain laiteux et grain pâteux mou). L'expérience a été répétée pendant trois ans.

L'augmentation de la fertilisation azotée permet d'accroître le rendement en matière sèche (MS) du blé seulement, alors qu'elle cause une augmentation linéaire du pourcentage de matières azotées (MAT) et du rendement en MAT par unité de surface des quatre céréales.

L'effet du stade de récolte sur le rendement en MS des céréales est hautement significatif. Comparativement au stade gonflement, lorsque l'avoine est récoltée au stade épiaison, grain laiteux et grain pâteux mou, le rendement augmente de 167,243 et $351 \%$, respectivement. Ces augmentations sont de 135 , 265 et $319 \%$ pour le blé ; 161,274 et
$338 \%$ pour l'orge ; et 165,458 et $517 \%$ pour le triticale.

La teneur en MAT de l'avoine est de $17,0^{\mathrm{a}}$; $13,3^{\mathrm{b}} ; 10,7^{\mathrm{c}}$ et $8,3^{\mathrm{d}} \%$ aux quatre stades étudiés. Ces valeurs sont respectivement, de $18,5^{\mathrm{a}} ; 14,9^{\mathrm{b}} ; 10,1^{\mathrm{c}}$ et $8,9^{\mathrm{d}} \%$ pour le blé $19,8^{a} ; 15,2^{\mathrm{b}} ; 11,6^{\mathrm{c}}$ et $10,1 \mathrm{~d} \%$ pour l'orge ; et $21,1^{a} ; 17,2^{b} ; 9,3^{c}$ et $8,8^{d} \%$ pour le triticale. La digestibilité in vitro de la MS aux quatre stades étudiés est de $76,3^{a} ; 69,5^{b} ; 58,6^{c}$ et $57,6^{d} \%$, respectivement, pour l'avoine; $74,8^{\mathrm{a}} ; 68,8^{\mathrm{b}}$; $62,5^{\circ}$ et $62,1^{\circ} \%$ pour le blé ; $76,4^{\mathrm{a}} ; 66,8^{\mathrm{b}}$; $63,7^{c}$ et $63,4^{c} \%$ pour l'orge ; et $78,3^{a} ; 73,3^{b}$; $64,5^{c}$ et $62,9^{d} \%$ pour le triticale.

En conclusion, une coupe tardive des céréales se traduit par une augmentation du rendement en MS mais aussi par une diminution de la teneur en MAT, de la digestibilité in vitro de la MS et, par le fait même, de la valeur nutritive du fourrage. Une coupe effectuée au stade épiaison et une fertilisation azotée d'environ $100 \mathrm{~kg} / \mathrm{ha}$ seraient un bon compromis pour obtenir une quantité et une qualité acceptables des céréales plantes entières. 